Article

\title{
Extended Fuzzy Metrics and Fixed Point Theorems
}

\author{
Valentín Gregori $^{1}{ }^{[}$, Juan-José Miñana ${ }^{2, *}{ }^{\circledR}$ and David Miravet ${ }^{1}$ \\ 1 Instituto de Investigación para la Gestión Integrada de Zonas Costeras, Universitat Politècnica de València, \\ Campus de Gandia, Calle Paranimf 1, 46730 Gandia, Spain; vgregori@mat.upv.es (V.G.); \\ damifor@alumni.uv.es (D.M.) \\ 2 Departament de Ciències Matemàtiques i Informàtica, Universitat de les Illes Balears, \\ Carretera de Valldemossa km 7.5, 07122 Palma, Spain \\ * Correspondence: jj.minana@uib.es; Tel.: +34-971-172901
}

Received: 31 December 2018; Accepted: 20 March 2019; Published: 25 March 2019

check for updates

\begin{abstract}
In this paper, we study those fuzzy metrics $M$ on $X$, in the George and Veeramani's sense, such that $\bigwedge_{t>0} M(x, y, t)>0$. The continuous extension $M^{0}$ of $M$ to $X^{2} \times[0,+\infty$ [ is called extended fuzzy metric. We prove that $M^{0}$ generates a metrizable topology on $X$, which can be described in a similar way to a classical metric. $M^{0}$ can be used for simplifying or improving questions concerning $M$; in particular, we expose the interest of this kind of fuzzy metrics to obtain generalizations of fixed point theorems given in fuzzy metric spaces.
\end{abstract}

Keywords: fuzzy metric space; fuzzy contractive mapping; fixed point

MSC: 54A40; 54D35; 54E50

\section{Introduction}

In 1975, Kramosil and Michalek introduced in [1] a notion of fuzzy metric space. Later, George and Veeramani in [2] strengthened some conditions on this concept. According to [2], a fuzzy metric space is an ordered triple $(X, M, *)$ such that $X$ is a (non-empty) set, $*$ is a continuous $t$-norm and $M$ is a fuzzy set on $X \times X \times] 0,+\infty[$ satisfying the following conditions, for all $x, y, z \in X, s, t>0$ :

(GV1) $M(x, y, t)>0$;

(GV2) $M(x, y, t)=1$ if and only if $x=y$;

(GV3) $M(x, y, t)=M(y, x, t)$;

(GV4) $M(x, y, t) * M(y, z, s) \leq M(x, z, t+s)$;

(GV5) $\left.\left.M_{x, y}:\right] 0,+\infty[\rightarrow] 0,1\right]$ is continuous, where $M_{x, y}(t)=M(x, y, t)$.

In such a case, we will say that $(M, *)$, or simply $M$, is a fuzzy metric on $X$.

George and Veeramani studied some aspects of the above concept in [2]. In particular, they proved that every fuzzy metric $M$ on $X$ generates a topology $\tau_{M}$ on $X$. It has as a base the family of open sets given by $\left\{B_{M}(x, r, t): x \in X, 0<r<1, t>0\right\}$, where $B_{M}(x, r, t)=\{y \in X: M(x, y, t)>1-r\}$ for all $x \in X, r \in] 0,1[$ and $t>0$. Then, different authors have contributed to study the topological properties of fuzzy metric spaces. For instance, in [3,4], it was proved that the class of topological spaces which are fuzzy metrizable coincides with the class of metrizable spaces.

A significant characteristic of a fuzzy metric is that it contains in its definition a parameter $t$. Related to it, we focus our attention in two facts about $M$. First, axiom (GV1) demands that $M(x, y, t)>0$ for all $x, y \in X$ and $t>0$, which is in accordance with classical metrics that do not take the value $+\infty$. Second, axiom (GV5) requires that $\left.\left.M_{x, y}:\right] 0,+\infty[\rightarrow] 0,1\right]$ be a continuous function, where $M_{x, y}(t)=M(x, y, t)$. These two facts suggest the introduction of a new notion of fuzzy metric in the next. 
Recall that a fuzzy metric $M$ is called stationary [5] if it does not depend on $t$ (in this case, we can write, simply, $M(x, y)$ ). Obviously, stationary fuzzy metrics are the closest to classical ones. In fact, if $M$ is a stationary fuzzy metric for the Lukasievicz $t$-norm, then $d=1-M$ is a metric on $X$, and $\tau_{M}$ agrees with $\tau_{d}$ (the topology generated by $d$ on $X$ ). It is clear that the expression of a stationary fuzzy metric $M$ on $X$ can be regarded as a fuzzy set $M^{0}$ on $X^{2} \times\left[0,+\infty\right.$ [ given by $M^{0}(x, y, t)=M(x, y)$ satisfying the two facts aforementioned, which are $M^{0}(x, y, t)>0$ and $M_{x, y}^{0}:[0,+\infty[\rightarrow] 0,1]$ is continuous for $x, y \in X$ and $t \geq 0$.

The above paragraph suggests to consider fuzzy sets $M^{0}: X^{2} \times[0,+\infty[\rightarrow] 0,1]$ satisfying all axioms of a fuzzy metric, but currently for $t \geq 0$. The term $\left(X, M^{0}, *\right)$ will be called extended fuzzy metric space (see Definition 1). The aim of this paper is the study of these spaces. The topics in which we will focus our attention will be: topology, completeness and contractivity with applications to fixed point theory.

First, we are interested in the relationship between fuzzy metrics and extended fuzzy metrics. Theorem 1 shows that every extended fuzzy metric $M^{0}$ is a natural extension of those fuzzy metrics, called extendable, which satisfy that $\bigwedge_{t>0} M(x, y, t)>0$, and vice versa. The natural extension is $M^{0}(x, y, 0)=\bigwedge_{t>0} M(x, y, t)$, for all $x, y \in X$. It is now a natural process to adapt the concepts of fuzzy metrics to extended fuzzy metrics. In particular, we introduce and generalize the concept of open ball $B_{M^{0}}(x, r, t)$ (see Section 3) and then we prove that the family $\left\{B_{M^{0}}(x, r, 0): x \in X, r \in\right] 0,1[\}$ is a base for a topology $\tau_{M^{0}}$ on $X$ finer than $\tau_{M}$, which is called the topology generated by $M^{0}$. This result is obtained after observing that $\left(N_{M}, *\right)$ is a stationary fuzzy metric on $X$, where $N_{M}(x, y)=$ $\bigwedge_{t>0} M(x, y, t)$ for all $x, y, \in X$. Then, it is easy to conclude (Proposition 3) that $\tau_{M^{0}}=\tau_{N_{M}}$ and hence $\tau_{M^{0}}$ is metrizable. Furthermore, we pointed out that a sequence $\left\{x_{n}\right\}$ converges to $x_{0}$ in $\tau_{M^{0}}$ if and only if $\lim _{n} M^{0}\left(x_{n}, x_{0}, 0\right)=1$. From the topological point of view (see Remark 3), the class of extended fuzzy metrics $\left(X, M^{0}, *\right)$ are so close to metrics that topological results related to $M^{0}$ can be established as a simple extension of classical concepts to the fuzzy setting, only by modifying the notation (which is left to the reader). Moreover, after proving that $M^{0}: X^{2} \times[0,+\infty[\rightarrow] 0,1]$ is continuous (see Proposition 4), we characterize those extendable fuzzy metrics in which $\tau_{M}=\tau_{M^{0}}$. Such spaces are the so called s-fuzzy metrics. In addition, this characterization motivates a study in the relationship between $\tau_{M^{0} \text {-convergence and s-convergence. }}$

With respect to completeness, although there are many concepts of Cauchy sequence in the literature (see [6]), we only pay attention to the original concept of Cauchy sequence given by $\mathrm{H}$. Sherwood in $P M$-spaces [7]. It was adapted later by George and Veeramani to the fuzzy metric context. According to [2], a sequence $\left\{x_{n}\right\}$ in a fuzzy metric space $(X, M, *)$ is said to be $M$-Cauchy, or simply Cauchy, if for each $\varepsilon \in] 0,1\left[\right.$ and each $t>0$ there exists $n_{0} \in \mathbb{N}$ such that $M\left(x_{n}, x_{m}, t\right)>1-\varepsilon$ for all $n, m \geq n_{0}$ or, equivalently, $\lim _{n, m} M\left(x_{n}, x_{m}, t\right)=1$ for all $t>0$. As usual, $X$ is said to be complete if every Cauchy sequence in $X$ is convergent with respect to $\tau_{M}$. In such a case, $M$ is also said to be complete. Then, an adaptation to the extended context of both aforementioned concepts is given. Furthermore, we provide some properties and observations on $M^{0}$-Cauchyness and $M^{0}$-completeness.

The last topic approached is contractivity, which plays a crucial role in fixed point theory. It should be expected that, in fuzzy metrics with strong properties, one should be able to weaken the usual contractive conditions in order to ensure the existence of fixed points, for a larger class of contractive mappings. Indeed, this is so. First, we notice that the condition itself of being $M$ extendable is used explicitly (Theorem 3.3 [8]), or, in a concealed or relaxed way, in order to obtain fixed point theorems (see Theorem 3.2 of [9] or Theorem 2.4 of [10]). We here go further and we will give a notion of $\psi$-0-contractive mapping (Definition 7), that is, contractivity assumed only at $t=0$. Then, we prove that there are $\psi$-0-contractive mappings in $\left(X, M^{0}, *\right)$ which are not $\psi$-contractive in $(X, M, *)$. Then, mimicking arguments in the literature, one can give fixed point theorems for extendable fuzzy metrics in a more general version. It is the case of Theorem 4 . The reader can find in this example a method for obtaining more general results in fixed point theory, but for extendable fuzzy metrics. Several examples illustrate the theory through the paper. 
The structure of the paper is as follows. In Section 2, we introduce and study the concept of extended fuzzy metric. Section 3 is devoted to establish a topology from an extended fuzzy metric and to characterize convergent sequences in it. Section 4 approaches the relationship between $s$-convergence

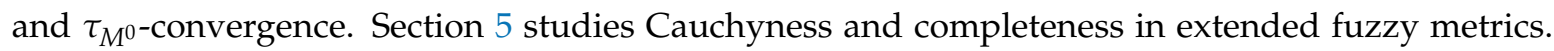
Section 6 is dedicated to contractivity and fixed point theorems.

\section{Extended Fuzzy Metrics}

We begin this section introducing the announced concept of extended fuzzy metric space.

Definition 1. The term $\left(X, M^{0}, *\right)$ is called an extended fuzzy metric space if $X$ is a (non-empty) set, * is a continuous $t$-norm and $M^{0}$ is a fuzzy set on $X^{2} \times[0,+\infty$ [ satisfying the following conditions, for each $x, y, z \in X$ and $t, s \geq 0$

(EFM1) $M^{0}(x, y, t)>0$;

(EFM2) $M^{0}(x, y, t)=1$ if and only if $x=y$;

(EFM3) $M^{0}(x, y, t)=M^{0}(y, x, t)$;

(EFM4) $M^{0}(x, y, t) * M^{0}(y, z, s) \leq M^{0}(x, z, t+s)$;

(EFM5) $M_{x, y}^{0}:[0,+\infty[\rightarrow] 0,1]$ is continuous, where $M_{x, y}^{0}(t)=M^{0}(x, y, t)$.

It is also said that $\left(M^{0}, *\right)$, or simply $M^{0}$, is an extended fuzzy metric on $X$. If $\star$ is a continuous $t$-norm satisfying $\star \leq *$, then $\left(M^{0}, \star\right)$ is also an extended fuzzy metric on $X$.

Remark 1. Recently, in [11], it was introduced the concept of extended fuzzy b-metric space, with the aim of generalizing the notion of fuzzy b-metric space. Both notions generalize the concept of fuzzy metric by means of relaxing the triangle inequality. Nevertheless, the goal of introducing Definition 1 is to "extend" in the concept of fuzzy metric, given by George and Veeramani, the domain of definition of the t parameter to $[0,+\infty[$. Thus, extended fuzzy b-metrics are not related to the new concept introduced above.

After introducing this new concept, we present some examples of it.

\section{Example 1.}

(a) If $M$ is a stationary fuzzy metric on $X$, then $M^{0}(x, y, t)=M(x, y)$ for all $x, y \in X$ and $t \geq 0$ is obviously an extended fuzzy metric on $X$, for the same $t$-norm. Since, again, $t$ does not play any role in the definition of $M^{0}$, we also say that $M^{0}$ is stationary. Furthermore, since, in this case, the expression of $M$ can be regarded itself as an extended fuzzy metric on $X$, we will not distinguish between $M$ and $M^{0}$, if confusion is not possible.

(b) Let $X$ be $] 0,+\infty\left[\right.$ and define the fuzzy set $M^{0}$ on $X^{2} \times\left[0,+\infty\left[\right.\right.$ given by $M^{0}(x, y, t)=\frac{\min \{x, y\}+t}{\max \{x, y\}+t}$. Then, $M^{0}$ is an extended fuzzy metric on $X$, for the product $t$-norm.

(c) Let $(X, d)$ be a metric space and $\phi:[0,+\infty[\rightarrow] 0,1]$ a non-decreasing continuous function with $\phi(0)>0$. Then, $\left(X, M_{\phi}^{0}, *\right)$ is an extended fuzzy metric, where $*$ is the product $t$-norm and $M_{\phi}^{0}(x, y, t)=\frac{\phi(t)}{\phi(t)+d(x, y)}$, for all $x, y \in X$ and $t \geq 0$.

The following theorem shows the relationship between fuzzy metrics and extended fuzzy metrics that one can observe in the last example.

Theorem 1. Let $M$ be a fuzzy set on $\left.X^{2} \times\right] 0,+\infty\left[\right.$, and denote by $M^{0}$ its extension to $X^{2} \times[0,+\infty[$ given by $M^{0}(x, y, t)=M(x, y, t)$ for all $x, y \in X, t>0$, and $M^{0}(x, y, 0)=\bigwedge_{t>0} M(x, y, t)$. Then, $\left(X, M^{0}, *\right)$ is an extended fuzzy metric space if and only if $(X, M, *)$ is a fuzzy metric space satisfying for each $x, y \in X$ the condition $\bigwedge_{t>0} M(x, y, t)>0$.

Proof. Suppose that $\left(M^{0}, *\right)$ is an extended fuzzy metric on $X$. Then, clearly, $(M, *)$ is a fuzzy metric on $X$. Now, we will see that $\bigwedge_{t>0} M(x, y, t)>0$ for all $x, y \in X$. 
Take $x, y \in X$. Since $M_{x, y}$ is not decreasing on $] 0,+\infty\left[\right.$ and $M_{x, y}^{0}$ is continuous at $t=0$, then

$$
\bigwedge_{t>0} M(x, y, t)=\lim _{t \rightarrow 0} M(x, y, t)=\lim _{t \rightarrow 0} M_{x, y}^{0}(t)=M_{x, y}^{0}(0)=M^{0}(x, y, 0)>0 .
$$

Conversely, let $(X, M, *)$ be a fuzzy metric space satisfying $\bigwedge_{t>0} M(x, y, t)>0$ for each $x, y \in X$. Attending to the hypothesis and by construction of $M^{0}$, we have that (EFM1) and (EFM3) are fulfilled. We will show the rest of the axioms.

(EFM2) Suppose $M^{0}(x, y, t)=1$ for some $t>0$. Then, $M(x, y, t)=1$ and so $x=y$. If $M^{0}(x, y, 0)=$ 1 , then $\bigwedge_{t>0} M(x, y, t)=1$ and thus $M(x, y, t)=1$ for all $t>0$, and so $x=y$. Obviously, if $x=y$, then $M^{0}(x, y, t)=1$ for all $t \geq 0$.

(EFM4) Let $x, y, z \in X$. We will distinguish three possibilities on $t, s \geq 0$.

1. If $t, s>0$, then (EFM4) is fulfilled since $M$ is a fuzzy metric.

2. Suppose $t>0$ and $s=0$ (the case $t=0$ and $s>0$ is analogous). Then, for $\varepsilon \in] 0, t$, we have that

$$
M^{0}(x, z, t+0)=M^{0}(x, z, t)=M(x, z, t) \geq M(x, y, t-\varepsilon) * M(y, z, \varepsilon) .
$$

Then, taking limits as $\varepsilon$ tends to 0 in the last inequality, we obtain

$$
\begin{gathered}
M^{0}(x, z, t+0) \geq \lim _{\varepsilon \rightarrow 0}(M(x, y, t-\varepsilon) * M(y, z, \varepsilon))= \\
=\left(\lim _{\varepsilon \rightarrow 0} M(x, y, t-\varepsilon)\right) *\left(\lim _{\varepsilon \rightarrow 0} M(y, z, \varepsilon)\right)= \\
=M(x, y, t) *\left(\bigwedge_{\varepsilon>0} M(y, z, \varepsilon)\right)=M^{0}(x, y, t) * M^{0}(y, z, 0) .
\end{gathered}
$$

3. Suppose $t=s=0$. Then, we have that

$$
\begin{gathered}
M^{0}(x, z, 0+0)=M^{0}(x, z, 0)=\bigwedge_{t>0} M(x, z, t)=\lim _{t \rightarrow 0} M(x, z, t) \geq \\
\geq \lim _{t \rightarrow 0}(M(x, y, t / 2) * M(y, z, t / 2))=\left(\lim _{t \rightarrow 0} M(x, y, t / 2)\right) *\left(\lim _{t \rightarrow 0} M(y, z, t / 2)\right)= \\
=\left(\bigwedge_{t>0} M(x, y, t)\right) *\left(\bigwedge_{t>0} M(y, z, t)\right)=M^{0}(x, y, 0) * M^{0}(y, z, 0) .
\end{gathered}
$$

(EFM5) Since $M_{x, y}$ is continuous on $] 0,+\infty[$, and $] 0,+\infty[$ is open in $[0,+\infty[$, with the usual topology of $\mathbb{R}$ restricted to $\left[0,+\infty\left[\right.\right.$, then $M_{x, y}^{0}$ is continuous at each point of $] 0,+\infty$ [ for each $x, y \in X$. For $t=0$, we have that

$$
\lim _{t \rightarrow 0} M^{0}(x, y, t)=\lim _{t \rightarrow 0} M(x, y, t)=\bigwedge_{t>0} M(x, y, t)=M^{0}(x, y, 0)
$$

and so $M_{x, y}^{0}$ is continuous at $t=0$.

Hence, $\left(X, M^{0}, *\right)$ is an extended fuzzy metric space.

An immediate consequence of the preceding result is that, given an extended fuzzy metric space $\left(X, M^{0}, *\right)$, then $M_{x, y}^{0}:[0,+\infty[\rightarrow] 0,1]$ is a non-decreasing continuous function satisfying $M_{x, y}^{0}(0)=\bigwedge_{t>0} M^{0}(x, y, t)$, for all $x, y \in X$. Furthermore, we can deduce the following result proved by Gregori et al. in [8]. 
Proposition 1. Let $(X, M, *)$ be a fuzzy metric space. Define

$$
N_{M}(x, y)=\bigwedge_{t>0} M(x, y, t)
$$

Then, $\left(N_{M}, *\right)$ is a stationary fuzzy metric on $X$ if and only if $\bigwedge_{t>0} M(x, y, t)>0$ for all $x, y \in X$.

Theorem 1 motivates the introduction of the following definition.

Definition 2. Let $(X, M, *)$ be a fuzzy metric space. $M$ is called extendable if for each $x, y \in X$ the condition $\bigwedge_{t>0} M(x, y, t)>0$ is satisfied. In such a case, we will say that $M^{0}$ is the (fuzzy metric) extension of $M$, and that $M$ is the restriction of $M^{0}$.

From now on, by $N_{M}$, we are referring to the stationary fuzzy metric defined in Proposition 1 , whenever $(X, M, *)$ is an extendable fuzzy metric space. Thus, by the aforementioned proposition, we have that $M$ is extendable if and only if $\left(N_{M}, *\right)$ is a stationary fuzzy metric on $X$. In addition, by Theorem 1 , we have that $M$ is extendable if and only if $\left(X, M^{0}, *\right)$ is an extended fuzzy metric space, where $M^{0}$ is given by

$$
M^{0}(x, y, t)= \begin{cases}N_{M}(x, y), & t=0 \\ M(x, y, t), & t>0\end{cases}
$$

We continue our study providing an example of a non-extendable fuzzy metric. First, recall that, given a metric space $(X, d)$, if we define a function $M_{d}$ on $\left.X \times X \times\right] 0,+\infty[$ as follows

$$
M_{d}(x, y, t)=\frac{t}{t+d(x, y)}
$$

then $\left(X, M_{d}, \wedge\right)$ is a fuzzy metric space (see [2]) and $M_{d}$ is called the standard fuzzy metric induced by $d$. Furthermore, the topology $\tau_{M_{d}}$ coincides with the topology $\tau(d)$ on $X$ deduced from $d$.

Now, we are able to present the announced example of a non-extendable fuzzy metric.

Example 2. Let $(X, d)$ be a metric space where $X$ has at least two points. Then, the standard fuzzy metric $\left(X, M_{d}, \wedge\right)$ is not extendable. Indeed, given $x, y \in X$ such that $x \neq y$, then $d(x, y) \neq 0$ and so

$$
\bigwedge_{t>0} M_{d}(x, y, t)=\lim _{t \rightarrow 0} \frac{t}{t+d(x, y)}=0 .
$$

Remark 2. In the following, we will associate to an extendable fuzzy metric $M$ the extended fuzzy metric $M^{0}$ and the stationary fuzzy metric $N_{M}$, defined above. Then, it is satisfied $M^{0}(x, y, 0)=\bigwedge_{t>0} M(x, y, t)=N_{M}(x, y)$ for all $x, y \in X$. In an analogous way, we will associate $M$ and $N_{M}$ to an extended fuzzy metric $M^{0}$.

Notice that, due to the continuity of the real function $M_{x, y}^{0}$ on $\left[0,+\infty\left[\right.\right.$, whenever $M^{0}$ is an extended fuzzy metric, then the extension $M^{0}$ of an extendable fuzzy metric $M$, is unique.

\section{Topology Deduced from an Extended Fuzzy Metric}

In this section, we justify that we can define a topology from an extended fuzzy metric. We proceed in a similar way as in the fuzzy metric case. Thus, we begin defining a ball, both open and closed.

Let $\left(X, M^{0}, *\right)$ be an extended fuzzy metric space. For $\left.x \in X, r \in\right] 0,1[$ and $t \geq 0$, by analogy with fuzzy metric spaces, we define the open ball of center $x$, radius $r$ and parameter $t$ as

$$
B_{M^{0}}(x, r, t)=\left\{y \in X: M^{0}(x, y, t)>1-r\right\}
$$


In an analogous way, the closed ball is

$$
B_{M^{0}}[x, r, t]=\left\{y \in X: M^{0}(x, y, t) \geq 1-r\right\} .
$$

Clearly, for $t>0$, we have that $B_{M^{0}}(x, y, t)=B_{M}(x, y, t)$ and $B_{M^{0}}[x, y, t]=B_{M}[x, y, t]$. In addition, $B_{M^{0}}(x, y, 0)=B_{N_{M}}(x, y)$ and $B_{M^{0}}[x, y, 0]=B_{N_{M}}[x, y]$.

Notice that, for all $x \in X, t>0, r \in] 0,1\left[\right.$, we have that $B_{M^{0}}(x, r, 0) \subset B_{M}(x, r, t)$ and $B_{M^{0}}[x, r, 0] \subset B_{M}[x, r, t]$.

Let $\left(X, M^{0}, *\right)$ be an extended fuzzy metric space. It is well known that the family $\left\{B_{N_{M}}(x, r): x \in X, r \in\right] 0,1[\}$ is a base of the topology $\tau_{N_{M}}$ on $X$ deduced from the stationary fuzzy metric $N_{M}$. Thus, the family given by $\left\{B_{M^{0}}(x, r, 0): x \in X, r \in\right] 0,1[\}$ is a base for the topology $\tau_{N_{M}}$ on $X$, which will be denoted $\tau_{M^{0}}$, and it will be called deduced from $M^{0}$. Clearly, the open balls $B_{M^{0}}(x, y, 0)$ are $\tau_{M^{0}}$-open and the closed balls are $\tau_{M^{0}}$-closed. Obviously, $\tau_{M^{0}}$ is metrizable.

Moreover, since for all $x \in X, r \in] 0,1\left[, t>0\right.$ we have that $B_{M^{0}}(x, r, 0) \subseteq B_{M}(x, r, t)$, then it is obvious that $\tau_{M^{0}} \succeq \tau_{M}$. Consequently, the open balls $B_{M}(x, r, t)$ (for $t>0$ ) are $\tau_{M^{0}}$-open, and the closed balls $B_{M}[x, r, t]$ (for $t>0$ ) are $\tau_{M^{0}}$-closed.

Now, we focus on convergence of sequences in $\tau_{M^{0}}$. Recall that George and Veeramani characterized $\tau_{M}$-convergent sequences with the following result (see [2]).

Proposition 2. Let $(X, M, *)$ be a fuzzy metric space. A sequence $\left\{x_{n}\right\}$ in $X \tau_{M}$-converges to $x \in X$ if and only if $\lim _{n} M\left(x_{n}, x, t\right)=1$, for all $t>0$.

On account of the above considerations, we provide the next characterization for the extended fuzzy metric case.

Proposition 3. Let $\left(X, M^{0}, *\right)$ be an extended fuzzy metric space. A sequence $\left\{x_{n}\right\}$ in $X \tau_{M^{0}}$-converges to $x_{0}$ if and only if $\lim _{n} M^{0}\left(x_{n}, x_{0}, 0\right)=1$.

Proof. Since $\tau_{N_{M}}=\tau_{M^{0}}$, then a sequence $\left\{x_{n}\right\}$ in $X \tau_{M^{0}}$-converges to $x_{0} \in X$ if and only if $\left\{x_{n}\right\}$ $\tau_{N_{M}}$-converges to $x_{0}$. By Proposition 2, we have that $\left\{x_{n}\right\} \tau_{N_{M}}$-converges to $x_{0}$ if and only if

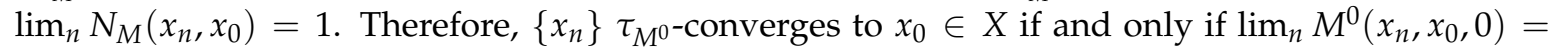
$\lim _{n} N_{M}\left(x_{n}, x_{0}\right)=1$, as we claimed.

Remark 3. On account of the exposed in this section, one can observe the similarity between extended fuzzy metrics and classical metrics, from the topological point of view. Indeed, in an extended fuzzy metric, the parameter $t$ does not play any role in the topological concepts. Nevertheless, such parameter remains being essential in different "metric" concepts for extendable fuzzy metrics, as we will see in the rest of the paper.

\section{Relationship between $\tau_{M^{0}}$-Convergence and s-Convergence in Extendable Fuzzy Metrics}

In this section, we compare $\tau_{M^{0}}$-convergent sequences with s-convergent ones, a stronger concept of convergence introduced in [8]. Such comparison is framed in the class of extendable fuzzy metrics. Recall that a sequence $\left\{x_{n}\right\}$ in a fuzzy metric space $(X, M, *)$ is called $s$-convergent to $x_{0} \in X$ if $\lim _{n} M\left(x_{n}, x_{0}, 1 / n\right)=1$. This concept of convergence motivated the authors in [8] to introduce a new class of fuzzy metrics, the so-called s-fuzzy metrics. Following [8], a fuzzy metric $M$ on $X$ is called $s$-fuzzy metric if every $\tau_{M}$-convergent sequence is s-convergent.

In order to fulfil the aforementioned main goal of this section, we begin showing the next proposition.

Proposition 4. Let $\left(X, M^{0}, *\right)$ be an extended fuzzy metric space. Then, $M^{0}$ is continuous with respect to the product topology, where $X$ is endowed with $\tau_{M^{0}}$ and $[0,+\infty[$ with the usual topology of $\mathbb{R}$ restricted to it. 
Proof. Let $\left\{\left(x_{n}, y_{n}, t_{n}\right)\right\}$ be a sequence in $X^{2} \times[0, \infty[$ which converges to $(x, y, t) \in[0, \infty[$ with respect to the product topology, where $X$ is endowed with $\tau_{M^{0}}$ and $[0,+\infty[$ with the usual topology of $\mathbb{R}$ restricted to it.

We will prove that $\lim _{n} M^{0}\left(x_{n}, y_{n}, t_{n}\right)=M^{0}(x, y, t)$ (i.e., $\left\{\left(x_{n}, y_{n}, t_{n}\right)\right\}$ converges to $M^{0}(x, y, t)$ in the usual topology of $\mathbb{R})$. To this end, we will distinguish two cases:

1. Suppose that $t>0$. Without loss of generality, we can suppose that $t_{n}>0$ for all $n \in \mathbb{N}$.

Following Proposition 1 of [12], if we consider the restriction $M$ of the extended fuzzy metric $M^{0}$, then $M$ is continuous on $\left.X^{2} \times\right] 0,+\infty[$ with respect to the product topology, where $X$ is endowed with $\tau_{M}$. Therefore,

$$
\lim _{n} M^{0}\left(x_{n}, y_{n}, t_{n}\right)=\lim _{n} M\left(x_{n}, y_{n}, t_{n}\right)=M(x, y, t)=M^{0}(x, y, t),
$$

and so $M^{0}$ is continuous at $(x, y, t)$.

2. Suppose now that $t=0$.

Let $\left\{\left(x_{n}, y_{n}, t_{n}\right)\right\}$ be a sequence that converges to $(x, y, 0) \in X^{2} \times\{0\}$. It follows that, for every $n \in \mathbb{N}$,

$$
M^{0}\left(x_{n}, y_{n}, t_{n}\right) \geq M^{0}\left(x_{n}, x, 0\right) * M^{0}\left(x, y, t_{n}\right) * M^{0}\left(y, y_{n}, 0\right)
$$

and

$$
M^{0}\left(x, y, t_{n}\right) \geq M^{0}\left(x, x_{n}, 0\right) * M^{0}\left(x_{n}, y_{n}, t_{n}\right) * M^{0}\left(y_{n}, y, 0\right)
$$

If we take limits on both equations and we use Proposition 3, we obtain the following expressions:

$$
\lim _{n} M^{0}\left(x_{n}, y_{n}, t_{n}\right) \geq \lim _{n} M^{0}\left(x, y, t_{n}\right),
$$

and

$$
\lim _{n} M^{0}\left(x, y, t_{n}\right) \geq \lim _{n} M^{0}\left(x_{n}, y_{n}, t_{n}\right)
$$

Now, by continuity of the function $M_{x, y}^{0}$, we have that $\lim _{n} M^{0}\left(x, y, t_{n}\right)=M^{0}(x, y, 0)$. We deduce that $\lim _{n} M^{0}\left(x_{n}, y_{n}, t_{n}\right)=M^{0}(x, y, 0)$ and so $M^{0}$ is continuous at $(x, y, 0)$.

Hence, $M^{0}$ is continuous at each point of $X^{2} \times\{0\}$.

The previous result is useful to prove the following proposition.

Proposition 5. Let $M$ be an extendable fuzzy metric on $X$. If $\left\{x_{n}\right\}$ is $\tau_{M^{0}}$-convergent to $x_{0}$, then $\lim _{n} M\left(x_{n}, x_{0}, 1 / n\right)=1$.

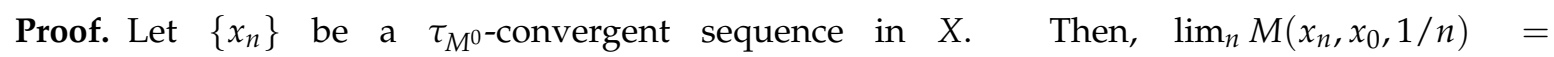
$\lim _{n} M^{0}\left(x_{n}, x_{0}, 1 / n\right)=M^{0}\left(x_{0}, x_{0}, 0\right)=1$, since $M^{0}$ is continuous and the sequence $\left\{\left(x_{n}, x_{0}, 1 / n\right)\right\}$ converges to $\left(x_{0}, x_{0}, 0\right)$ in the product topology.

The following example proves that the converse of the last proposition is false in general.

Example 3. (see Example 4.3 of [8]). Consider the extendable fuzzy metric space $(X, M, *)$, where $X=] 0,1]$, * is the Lukasievicz t-norm, and $M$ is given by

$$
M(x, y, t)=\left\{\begin{array}{cc}
1-\frac{1}{2} d(x, y)^{t}, & 0 \leq t \leq 1 \\
1-\frac{1}{2} d(x, y), & t>1
\end{array}\right.
$$


where $d$ is the usual metric of $\mathbb{R}$. The sequence $\left\{x_{n}\right\}$, where $x_{n}=1 / 2-1 / n^{n}$ for all $n \geq 2$, is $\tau_{M}$-convergent to $1 / 2$ since $\tau_{M}$ is the usual topology of $\mathbb{R}$ restricted to $\left.] 0,1\right]$. Moreover, $\lim _{n} M\left(x_{n}, 1 / 2,1 / n\right)=$ $\lim _{n}\left(1-\frac{1}{2}\left(\frac{1}{n^{n}}\right)^{1 / n}\right)=1$ and so $\left\{x_{n}\right\}$ is s-convergent. Now,

$$
N_{M}(x, y)=\bigwedge_{t>0} M(x, y, t)= \begin{cases}1, & \text { if } x=y \\ \frac{1}{2}, & \text { if } x \neq y\end{cases}
$$

Therefore, $\lim _{n} M^{0}\left(x_{n}, 1 / 2,0\right)=\lim _{n} N_{M}\left(x_{n}, 1 / 2\right)=1 / 2$ and so $\left\{x_{n}\right\}$ is not $\tau_{M^{0} \text {-convergent. }}$

On account of the above example, an interesting question is to characterize those extendable fuzzy metric spaces in which s-convergent sequences are $\tau_{M_{0}}$-convergent. We approach this problem in the rest of the section. To such goal, we begin expressing Theorem 4.2 of [8] in our context.

Theorem 2. Let $M$ be an extendable fuzzy metric on $X$. Then, $\tau_{M}=\tau_{M^{0}}$ if and only if $M$ is an s-fuzzy metric.

Thus, an immediate corollary of the previous theorem is the following one.

Corollary 1. Let $M$ be an extendable fuzzy metric on $X$. If $M$ is an s-fuzzy metric, then every s-convergent sequence is $\tau_{M^{0} \text {-convergent. }}$

Even more, the next theorem shows that the converse of the preceding corollary is also true.

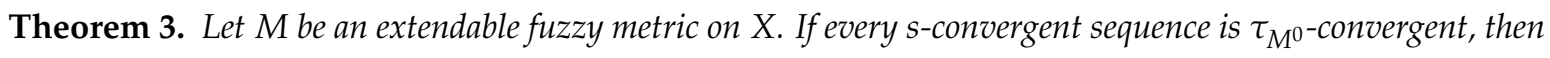
$M$ is an s-metric.

Proof. Let $M$ be an extendable fuzzy metric on $X$.

Suppose that $M$ is not an s-fuzzy metric. Then, there exists a $\tau_{M}$-convergent sequence $\left\{x_{n}\right\}$ to some $x_{0} \in X$, which is not s-convergent (to $\left.x_{0}\right)$ in $(X, M)$. We will construct an s-convergent subsequence $\left\{x_{n_{k}}\right\}$ of $\left\{x_{n}\right\}$, which is not $\tau_{M^{0}}$-convergent. To construct such subsequence, we are focused on two facts:

First, since $\left\{x_{n}\right\}$ is $\tau_{M}$-convergent, then, for each $\left.\varepsilon \in\right] 0,1\left[\right.$ and each $t>0$, we can find $n_{0} \in \mathbb{N}$ such that $M\left(x_{n}, x_{0}, t\right)>1-\varepsilon$ whenever $n \geq n_{0}$.

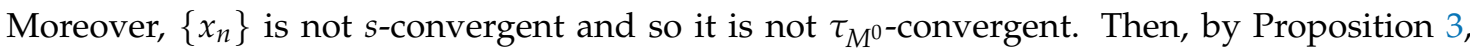
we have that there exists $\left.\varepsilon_{0} \in\right] 0,1\left[\right.$ such that, for each $k \in \mathbb{N}$, we can find $n_{k}>k$ such that $M^{0}\left(x_{n_{k}}, x_{0}, 0\right) \leq 1-\varepsilon_{0}$.

Fix $k=2$. On the one hand, if we consider $\varepsilon=t=\frac{1}{2}$, then there exists $n_{2}^{\prime} \in \mathbb{N}$ such that $M\left(x_{n}, x_{0}, \frac{1}{2}\right)>1-\frac{1}{2}$ whenever $n \geq n_{2}^{\prime}$. On the other hand, given $n_{2}^{\prime} \in \mathbb{N}$, we can find $n_{2}>n_{2}^{\prime}$ such that $M^{0}\left(x_{n_{2}}, x_{0}, 0\right) \leq 1-\varepsilon_{0}$.

From this element $x_{n_{2}}$, we construct inductively on $k \in \mathbb{N}$ the announced subsequence of $\left\{x_{n}\right\}$ as follows.

Take $k \in \mathbb{N}$. As before, there exists $n_{k}^{\prime} \in \mathbb{N}$, with $n_{k}^{\prime} \geq n_{k-1}$, such that $M\left(x_{n}, x_{0}, \frac{1}{k}\right)>1-\frac{1}{k}$ whenever $n \geq n_{k}^{\prime}$. Furthermore, given $n_{k}^{\prime} \in \mathbb{N}$, we can find $n_{k}>n_{k}^{\prime}$ such that $M^{0}\left(x_{n_{k}}, x_{0}, 0\right) \leq 1-\varepsilon_{0}$.

Therefore, the constructed subsequence $\left\{x_{n_{k}}\right\}$ satisfies the following properties:

$$
M\left(x_{n_{k}}, x_{0}, 1 / k\right)>1-1 / k
$$

and

$$
M^{0}\left(x_{n_{k}}, x_{0}, 0\right) \leq 1-\varepsilon_{0},
$$


for each $k \in \mathbb{N}$. Thus, taking limits in the above two inequalities, we have that

$$
\lim _{k} M\left(x_{n_{k}}, x_{0}, 1 / k\right)=1,
$$

and

$$
\lim _{k} M^{0}\left(x_{n_{k}}, x_{0}, 0\right) \leq 1-\varepsilon_{0} .
$$

Thus, $\left\{x_{n_{k}}\right\}$ is an s-convergent sequence that is not $\tau_{M^{0}}$-convergent and the proof is concluded.

As a consequence of Corollary 1 and Theorem 3, we can state the following result, which characterize $s$-fuzzy metrics whenever consider an extendable fuzzy metric.

Corollary 2. Let $M$ be an extendable fuzzy metric on $X$. Then, $M$ is an s-metric if and only if every s-convergent sequence is $\tau_{M^{0} \text {-convergent. }}$

\section{Cauchyness and Completeness}

This section is dedicated to study the completeness of extended fuzzy metric spaces. To this goal, we begin introducing a concept of Cauchy sequence in such spaces.

Definition 3. Let $\left(X, M^{0}, *\right)$ be an extended fuzzy metric space. A sequence $\left\{x_{n}\right\}$ in $X$ is called $M^{0}$-Cauchy if, given $\varepsilon \in] 0,1\left[\right.$, we can find $n_{\varepsilon} \in \mathbb{N}$ such that $M^{0}\left(x_{n}, x_{m}, 0\right)>1-\varepsilon$ for all $n, m \geq n_{\varepsilon}$. Then, $\left\{x_{n}\right\}$ is $M^{0}$-Cauchy if and only if $\lim _{m, n} M^{0}\left(x_{m}, x_{n}, 0\right)=1$.

Remark 4. From now on, we will say that a sequence is $M^{0}$-convergent instead of $\tau_{M^{0}}$-convergent in order to simplify the notation.

An immediate relationship between $M^{0}$-convergent sequences and $M^{0}$-Cauchy ones is next.

Proposition 6. Let $\left(X, M^{0}, *\right)$ be an extended fuzzy metric space. Every $M^{0}$-convergent sequence is $M^{0}$-Cauchy.

Proof. Suppose $\left\{x_{n}\right\}$ is $M^{0}$-convergent to $x_{0}$. The conclusion follows from the inequality $M^{0}\left(x_{m}, x_{n}, 0\right) \geq M^{0}\left(x_{m}, x_{0}, 0\right) * M^{0}\left(x_{0}, x_{n}, 0\right)$.

The $M^{0}$-Cauchy's concept is according to the idea of Cauchy sequence in [2], since it can be expressed as $\lim _{m, n} M^{0}\left(x_{m}, x_{n}, t\right)=1$ for all $t \geq 0$. Clearly, every $M^{0}$-Cauchy sequence is $M$-Cauchy, and the converse is false, in general, as the following example shows.

Example 4. Consider the extendable fuzzy metric space $(X, M, *)$, where $X=] 0,+\infty[, *$ is the product t-norm and $M$ is given by

$$
M(x, y, t)=\frac{\min \{x, y\}+t}{\max \{x, y\}+t}
$$

for each $x, y \in X$ and $t>0$.

The sequence $\left\{x_{n}\right\}$, where $x_{n}=\frac{1}{n}$ for each $n \in \mathbb{N}$, is M-Cauchy. Indeed, for each $t>0$, we have that

$$
\lim _{n, m} M\left(x_{n}, x_{m}, t\right)=\frac{\min \{1 / n, 1 / m\}+t}{\max \{1 / n, 1 / m\}+t}=1 .
$$

Nevertheless, $\left\{x_{n}\right\}$ is not $M_{0}$-Cauchy. Indeed, observe that

$$
M^{0}(x, y, 0)=\bigwedge_{t>0} M(x, y, t)=\bigwedge_{t>0} \frac{\min \{x, y\}+t}{\max \{x, y\}+t}=\frac{\min \{x, y\}}{\max \{x, y\}}
$$

for each $x, y \in X$. 
Then, if $\left\{x_{n}\right\}$ is $M^{0}$-Cauchy, we will have that

$$
\lim _{n, m} M^{0}\left(x_{n}, x_{m}, 0\right)=\lim _{n, m} \frac{\min \{1 / n, 1 / m\}}{\max \{1 / n, 1 / m\}}=1 .
$$

Now, if we consider $m=2 n$, then

$$
\lim _{n, m} \frac{\min \{1 / n, 1 / m\}}{\max \{1 / n, 1 / m\}}=\lim _{n}=\frac{1 / 2 n}{1 / n}=\frac{1}{2},
$$

a contradiction.

We continue introducing the following notion of completeness in a natural way.

Definition 4. An extended fuzzy metric space $\left(X, M^{0}, *\right)$ is called complete if every $M^{0}$-Cauchy sequence is $M^{0}$-convergent. It is also said that $M^{0}$ is complete, and, if confusion does not arise, that $X$ is $M^{0}$-complete.

An immediate property of the above definition is the next one.

Proposition 7. $\left(X, M^{0}, *\right)$ is complete if and only if $\left(X, N_{M}, *\right)$ is complete.

Proof. The sequence $\left\{x_{n}\right\}$ is $M^{0}$-Cauchy if and only if it is $N_{M}$-Cauchy. Then, the conclusion follows from the fact that $\tau_{M^{0}}=\tau_{N_{M}}$.

The next proposition shows the relationship between $M^{0}$-completeness and $M$-completeness, when we consider an extendable s-fuzzy metric.

Proposition 8. Let $M$ be an extendable s-fuzzy metric on $X$. If $(X, M, *)$ is complete, then $\left(X, M^{0}, *\right)$ is complete.

Proof. Let $(X, M, *)$ be a complete extendable $s$-fuzzy metric space and let $\left\{x_{n}\right\}$ be an $M^{0}$-Cauchy sequence in $X$. Then, $\left\{x_{n}\right\}$ is $M$-Cauchy and thus $\left\{x_{n}\right\}$ is $\tau_{M}$-convergent in $X$, since $M$ is complete. Now, by Theorem 2, we conclude that $\left\{x_{n}\right\}$ is $M^{0}$-convergent.

The converse of the last proposition is false, in general, as it is shown in the following example.

Example 5. Let $(X, M, *)$ be the extendable s-fuzzy metric space, where $X=] 0,+\infty[, M(x, y, t)=$ $\frac{\min \{x, y\}+t}{\max \{x, y\}+t}$ and $*$ is the $t$-norm product (see [8]).

We notice that $\tau_{M}$ is the usual topology of $\mathbb{R}$ restricted to $] 0,+\infty\left[\right.$. Furthermore, $\tau_{M^{0}}=\tau_{M}$ since $M$ is an s-fuzzy metric.

On the one hand, we have that $\left(X, N_{M}, *\right)$ is complete (see $\left.[13,14]\right)$, where $N_{M}$ is given by

$$
N_{M}(x, y)=M^{0}(x, y, 0)=\frac{\min \{x, y\}}{\max \{x, y\}} .
$$

We conclude, by Proposition 7 , that $\left(X, M^{0}, *\right)$ is complete.

On the other hand, $(X, M, *)$ is not complete, since $\{1 / n\}$ is an $M$-Cauchy sequence in $X$, but it does not converge for $\tau_{M}$.

To finish this section, we provide an example, which shows that, for every metrizable topological space, we can find a compatible non-stationary extended fuzzy metric which is an s-fuzzy metric. 
Example 6. Let $(X, \tau)$ be a metrizable topological space. Suppose that $d$ is a compatible metric on $X$, i.e., $\tau(d)=\tau$. Consider the extendable fuzzy metric $M_{\phi}(x, y, t)=\frac{\phi(t)}{\phi(t)+d(x, y)}$ of Example 1 (c). It is not hard to check that $\tau_{M_{\phi}}=\tau$.

If $\left\{x_{n}\right\}$ is $\tau_{M_{\phi}}$-convergent to $x_{0}$, then

$$
\lim _{n} M_{\phi}\left(x_{n}, x_{0}, 1 / n\right)=\lim _{n} \frac{\phi(1 / n)}{\phi(1 / n)+d\left(x_{n}, x_{0}\right)}=\frac{\phi(0)}{\phi(0)}=1 .
$$

Then, $M_{\phi}$ is an extendable s-fuzzy metric on $X$, and thus, by Theorem $2, \tau_{M_{\phi}^{0}}=\tau_{M_{\phi}}$.

\section{Contractivity and Fixed Point Theorems}

In this section, we show how it is possible to give a more general version of fixed point theorems in extendable fuzzy metrics $M$ by means of a weaker contractive condition (see Remark 5) on the extended fuzzy metric $M^{0}$. We begin recalling a notion of contractive mapping introduced by Mihet in [15]. It was adapted by Gregori and Miñana in [16] to the George and Veeramani context as follows.

Definition 5. Let $\Psi$ be the class of all mappings $\psi:] 0,1] \rightarrow] 0,1]$ such that $\psi$ is continuous, non-decreasing and $\psi(t)>t$ for all $t \in] 0,1[$. Let $\psi \in \Psi$. A mapping $f: X \rightarrow X$ is called fuzzy $\psi$-contractive mapping if:

$$
M(f(x), f(y), t) \geq \psi(M(x, y, t)) \text { for all } \mathrm{x}, \mathrm{y} \in \mathrm{X} \text { and } \mathrm{t}>0
$$

According to the above definition and extending the classical concept of a contractive sequence, then a sequence $\left\{x_{n}\right\}$ in $X$ is called $(f u z z y) \psi$-contractive sequence if it satisfies

$$
M\left(x_{n+1}, x_{n+2}, t\right) \geq \psi\left(M\left(x_{n}, x_{n+1}, t\right)\right) \text { for all } n \in \mathbb{N} \text { and } t>0 .
$$

An immediate consequence of the previous notion is the next proposition, whose proof is straightforward.

Proposition 9. Let $(X, M, *)$ be a fuzzy metric space. If $f: X \rightarrow X$ is $\psi$-contractive, then, for each $x_{0} \in X$, the sequence of iterates $\left\{x_{n}\right\}$ where $x_{1}=f\left(x_{0}\right), \ldots, x_{n}=f\left(x_{n-1}\right)$, for $n=2,3, \cdots$, is $\psi$-contractive.

We recall the concept of contractivity given by Gregori and Sapena in [17].

Definition 6. Let $(X, M, *)$ be a fuzzy metric space. A mapping $f: X \rightarrow X$ is called fuzzy contractive if there exists $k \in] 0,1[$ such that

$$
\frac{1}{M(f(x), f(y), t)}-1 \leq k\left(\frac{1}{M(x, y, t)}-1\right)
$$

for each $x, y \in X$ and $t>0$. ( $k$ is called the contractive constant of $f$.)

Mihet observed in [15] that a fuzzy contractive mapping is a fuzzy $\psi$-contractive one for $\psi(s)=$ $\frac{s}{s+k(1-s)}$ for each $\left.\left.s \in\right] 0,1\right]$.

As we have proceeded throughout the paper, we will adapt the notion of fuzzy $\psi$-contractive mapping to extended fuzzy metrics.

Definition 7. Let $(X, M, *)$ be an extendable fuzzy metric space. A mapping $f: X \rightarrow X$ is called fuzzy $\psi$ - $M^{0}$-contractive if Equation (1) is satisfied for all $t \geq 0$. Particularly, $f$ is called fuzzy $\psi$-0-contractive if Equation (1) is satisfied for $t=0$. 
Remark 5. Due to the continuity of $M_{x, y}^{0}$ for all $x, y \in X$, it is clear that $f$ is $\psi-M^{0}$-contractive if and only if $f$ is $\psi$-contractive. Nevertheless, the next example shows that the fuzzy $\psi$-0-contractive condition is weaker than the fuzzy $\psi-M^{0}$-contractive one.

Example 7. Consider $X=\mathbb{R}$ endowed with the usual metric $d$. Define the fuzzy set $M$ on $\left.X^{2} \times\right] 0,+\infty[$ as follows:

$$
M(x, y, t)=\frac{1+\alpha(t) d(x, y)}{1+d(x, y)}
$$

where $\alpha:\left[0,+\infty\left[\rightarrow\left[0,+\infty\left[\right.\right.\right.\right.$ is given by $\alpha(t)=\frac{t}{1+t}$ for all $t \geq 0$. Then, one can verify that $(X, M, *)$ is an extendable fuzzy metric space for the Lukasievicz $t$-norm. We will see that $f: \mathbb{R} \rightarrow \mathbb{R}$ given by $f(x)=x / 2$, for $x \in \mathbb{R}$ is fuzzy $\psi$-0-contractive.

Let $x, y \in X$. Then,

$$
\begin{gathered}
\frac{1}{M(f(x), f(y), 0)}-1=\left(\frac{1}{1+1 / 2|x-y|}\right)^{-1}-1=\frac{|x-y|}{2}=\frac{1}{2}(1+|x-y|-1)= \\
=\frac{1}{2}\left(\left(\frac{1}{1+|x-y|}\right)^{-1}-1\right)=\frac{1}{2}\left(\frac{1}{M(x, y, 0)}-1\right) .
\end{gathered}
$$

Then, attending to the above comment, $f$ is fuzzy $\psi$-0-contractive, for $\psi(s)=\frac{s}{s+k(1-s)}$ for each $\left.\left.s \in\right] 0,1\right]$. Now, we will see that $f$ is not fuzzy contractive. Indeed, if we take $t=1$, then, for $x, y \in \mathbb{R}$, we have

$$
\frac{1}{M(f(x), f(y), 1)}-1=\left(\frac{1+\frac{1}{2} \cdot \frac{1}{2}|x-y|}{1+\frac{1}{2}|x-y|}\right)^{-1}-1=\frac{|x-y|}{4+|x-y|} .
$$

On the other hand,

$$
k\left(\frac{1}{M(x, y, 1)}-1\right)=k\left(\left(\frac{1+\frac{1}{2}|x-y|}{1+|x-y|}\right)^{-1}-1\right)=k\left(\frac{|x-y|}{2+|x-y|}\right)
$$

In this case, the inequality

$$
\frac{1}{M(f(x), f(y), 1)}-1 \leq k\left(\frac{1}{M(x, y, 1)}-1\right)
$$

is not possible for $k \in[0,1[$. Indeed, for a fixed $y \in \mathbb{R}$, if $x$ tends to $+\infty$, then we obtain $1 \leq k$, a contradiction.

According to Definition 7, we introduce the notion of fuzzy $\psi$-0-contractive sequence.

Definition 8. Let $(X, M, *)$ be an extendable fuzzy metric space. A sequence $\left\{x_{n}\right\}$ of $X$ is called fuzzy $\psi$-0-contractive if there exists $\psi \in \Psi$ such that

$$
M^{0}\left(x_{n+1}, x_{n+2}, 0\right) \geq \psi\left(M^{0}\left(x_{n}, x_{n+1}, 0\right)\right) \quad \text { for all } n \in \mathbb{N} .
$$

The sequence of iterates constructed from a fuzzy $\psi$-0-contractive mapping satisfies the following stronger property.

Proposition 10. Let $(X, M, *)$ be an extendable fuzzy metric space, and let $f: X \rightarrow X$ be a fuzzy $\psi$-0-contractive mapping. Consider, for $x_{0} \in X$, the sequence $\left\{x_{n}\right\}$ of iterates $x_{1}=f\left(x_{0}\right), x_{n}=f\left(x_{n-1}\right)$, $n=2,3, \ldots$ Then,

$$
M^{0}\left(x_{n+1}, x_{m+1}, 0\right) \geq \psi\left(M^{0}\left(x_{n}, x_{m}, 0\right)\right.
$$


for every $n, m \in \mathbb{N}$.

Proof. It follows from the definition of fuzzy $\psi$-0-contractive function.

Mimicking the proof of Lemma 3.2 in [18] and using the preceding proposition, we obtain the following result.

Proposition 11. Let $\left(X, M^{0}, *\right)$ be an extended fuzzy metric space and let $f: X \rightarrow X$ be a fuzzy $\psi$-0-contractive mapping. Then, for each $x_{0} \in X$, the sequence of iterates $\left\{x_{n}\right\}$ is $M^{0}$-Cauchy.

As a consequence of the above result and following the arguments of [18], for instance, Corollary 3.9 of [18] admits the following more general version (see Remark 5).

Theorem 4. Let $(X, M, *)$ be an extendable complete fuzzy metric space. Then, every fuzzy $\psi$-0-contractive mapping $f: X \rightarrow X$ admits a unique fixed point.

\section{Conclusions}

In 1975, Kramosil and Michalek introduced a notion of fuzzy metric space in [1]. Then, it was slightly modified by George and Veeramani in [2]. Both notions share many properties. Indeed, they are topologically equivalent to classical metric spaces. Nevertheless, there exist metric properties for which the concept given by Kramosil and Michalek, and the one due to George and Veeramani differ. For instance, fuzzy metric spaces in the sense of Kramosil and Michalek are completable (as they are metric spaces), whereas the ones introduced by George and Veeramani do not.

A topic in which both notions of fuzzy metric aforementioned differ significantly with classical metrics is in fixed point theory. Indeed, the usual proofs of fixed point results in metric spaces do not work, in general, in fuzzy metric spaces. The inconvenience is due to the $t$-parameter and its relevance in the triangle inequality defined in a fuzzy metric space. In the last few years, several authors have contributed to the study of such topic, adapting classical fixed point theorems to the fuzzy context (see, for instance, $[9,10,18-21])$. The most of them must demand an extra condition on the fuzzy metric to get fixed point. Extended fuzzy metrics, introduced and studied in this paper, provide a notion that allows us to avoid the aforesaid inconvenience of the $t$-parameter. As it has been justified in Section 6, the significance of extended fuzzy metric spaces comes from the fact that, on them, we can relax the contractive condition and obtain a fixed point, without requiring any extra condition on the extended fuzzy metric space (see Theorem 4). Thus, extended fuzzy metric spaces can be considered as a prominent framework in the fuzzy metric context to the study of fixed point theory.

Author Contributions: V.G., J.-J.M. and D.M. have contributed equally to this work.

Funding: V. Gregori acknowledges the support of the Ministry of Economy and Competitiveness of Spain under Grant MTM2015-64373-P (MINECO/Feder, UE). Juan José Miñana acknowledges the partial support of the Spanish Ministry of Economy and Competitiveness under Grant TIN2016-81731-REDT (LODISCO II) and AEI/FEDER, UE funds, by the Programa Operatiu FEDER 2014-2020 de les Illes Balears, by project ref. PROCOE/4/2017 (Direcció General d'Innovació i Recerca, Govern de les Illes Balears), and by project ROBINS. The latter has received research funding from the EU H2020 framework under GA 779776. This publication reflects only the authors' views and the European Union is not liable for any use that may be made of the information contained therein.

Acknowledgments: The authors are very grateful to the reviewers for their valuable suggestions which have helped to improve the content of the paper.

Conflicts of Interest: The authors declare no conflict of interest.

\section{References}

1. Kramosil, K.; Michalek, J. Fuzzy metric and statistical metric spaces. Kybernetika 1975, 11, 336-344.

2. George, A.; Veeramani, P. On some results in fuzzy metric spaces. Fuzzy Set. Syst. 1994, 64, 395-399. [CrossRef] 
3. George, A.; Veeramani, P. Some theorems in fuzzy metric spaces. J. Fuzzy Math. 1995, 3, 933-940.

4. Gregori, V.; Romaguera, S. Some properties of fuzzy metric spaces. Fuzzy Set. Syst. 2000, 115, 485-489. [CrossRef]

5. Gregori, V.; Romaguera, S. Characterizing completable fuzzy metric spaces. Fuzzy Set. Syst. 2004, 144, 411-420. [CrossRef]

6. Gregori, V.; Miñana, J.J.; Morillas, S.; Sapena, A. Cauchyness and convergence in fuzzy metric spaces. RACSAM Rev. R. Acad. A 2017 111, 25-37. [CrossRef]

7. Sherwood, H. Complete probabilistic metric spaces. Probab. Theory Relat. Fields 1971, 20, 117-128. [CrossRef]

8. Gregori, V.; Miñana, J.J.; Morillas, S. A note on convergence in fuzzy metric spaces. Iran. J. Fuzzy Syst. 2014, 11, 75-85.

9. Wardowski, D. Fuzzy contractive mappings and fixed points in fuzzy metric spaces. Fuzzy Set. Syst. 2013, 222, 108-114. [CrossRef]

10. Mihet, D. A note on fuzzy contractive mappings in fuzzy metric spaces. Fuzzy Set. Syst. 2014, $251,83-91$. [CrossRef]

11. Mehmood, F.; Ali, R.; Ionescu, C.; Kamran, T. Extended fuzzy b-metric spaces. J. Math. Anal. 2017, 8, $124-131$.

12. Rodríguez-López, J.; Romaguera, S. The Hausdorff fuzzy metric on compact sets. Fuzzy Set. Syst. 2004, 147, 273-283. [CrossRef]

13. Gregori, V.; Miñana, J.J.; Morillas, S. Some questions in fuzzy metric spaces. Fuzzy Set. Syst. 2012, $204,71-85$. [CrossRef]

14. Radu, V. Some remarks on the probabilistic contractions on fuzzy Menger spaces. Automat. Comput. Appl. Math. 2002, 11, 125-131.

15. Mihet, D. Fuzzy $\psi$-contractive mappings in non-Archimedean fuzzy metric spaces. Fuzzy Set. Syst. 2008, 159, 739-744. [CrossRef]

16. Gregori, V.; Miñana, J.J. Std-convergence in fuzzy metric spaces. Fuzzy Set. Syst. 2015, 267, 140-143. [CrossRef]

17. Gregori, V.; Sapena, A. On fixed-point theorems in fuzzy metric spaces. Fuzzy Set. Syst. 2002, 125, $245-252$. [CrossRef]

18. Gregori, V.; Miñana, J.J. On fuzzy $\psi$-contractive sequences and fixed point theorems. Fuzzy Set. Syst. 2016, 300, 93-101. [CrossRef]

19. Chauhan, S.; Shatanawi, W.; Kumur, S.; Radenović, S. Existence and uniqueness of fixed points in modified intuitionistic fuzzy metric spaces. J. Nonlinear Sci. Appl. 2014, 7, 28-41. [CrossRef]

20. Došenović, T.; Rakić, D.; Carić, B.; Radenović, S. Multivalued generalizations of fixed point results in fuzzy metric spaces. Nonlinear Anal. Model. Control 2016, 21, 211-222.

21. Sedghi, S.; Shobkolaei, N.; Došenović, T.; Radenović, S. Suzuki-type of common fixed point theorems in fuzzy metric spaces. Math. Slovaca 2018, 68, 451-462. [CrossRef] 